\title{
Identification of transposon-tagged genes by the random sequencing of Mutator-tagged DNA fragments from Zea mays
}

\author{
Steven Hanley ${ }^{1}$, David Edwards ${ }^{1}$, David Stevenson ${ }^{1}$, Stephen Haines ${ }^{1}$, Matthew Hegarty ${ }^{1}$, Wolfgang Schuch ${ }^{2}$ and \\ Keith J. Edwards ${ }^{1, *}$ \\ ${ }^{1}$ IACR-Long Ashton Research Station, Department of Agricultural Sciences, University of Bristol, Long Ashton, Bristol \\ BS41 9AF, UK, and \\ ${ }^{2}$ Zeneca Wheat Improvement Centre, John Innes Centre, Norwich NR4 7UH, UK
}

Received 27 March 2000; revised 25 May 2000; accepted 8 June 2000.

*For correspondence (fax +44 1275394 281; e-mail keith.edwards@bbsrc.ac.uk).

\begin{abstract}
Summary
We have used a universal adaptor amplification procedure to isolate random Mutator-tagged fragments from Mutator-active maize plants. Direct sequence characterization of 761 Mutator-tagged fragments indicated that a significant number were homologous to sequences within the public databases. The ability of Mutator-tagged fragments to detect homology was not related to the length of the sequence within the range $100-400 \mathrm{bp}$. However, fragments above this size did show an increased chance of detecting homology to either expressed sequence tags or genes. Characterization of the insertion sites of the Mutator elements suggested that while it does target transcribed regions, Mutator does not appear to have any site preference within the transcription unit. Hybridization of previously unidentified Mutator-tagged fragments to arrayed cDNA libraries confirmed that many of these also showed homology to transcribed regions of the genome. Examination of back-crossed progeny confirmed that all the insertions examined were germinal; however, in all but one case, selfing five individual Mutatortagged lines failed to reveal an obvious phenotype. This study suggests that the random sequencing of Mutator-tagged fragments is capable of producing both a significant number of interesting transposon tagged genes and mutant plant lines, all of which could be extremely valuable in future gene discovery and functional genomics programmes.
\end{abstract}

Keywords: Mutator, transposon tagging, Zea mays, sequencing.

Introduction

The maize genome is relatively complex, presumably due to its ancient allotetraploid origins (Gaut and Doebley, 1997) and the presence of large numbers of both retroelements and transposons (Edwards etal., 1996; San Miguel etal., 1996). The number and variety of transposable elements within the maize genome have greatly facilitated its use in insertional mutagenesis programmes, whilst its allotetraploid-based gene redundancy has allowed the characterization of mutants which may be lethal in a diploid species. These features, which limit the use of maize as a model for cereal genome structure, make it an eminently suitable model for the study of cereal gene function (O'Sullivan et al., 2000).

Transposons were first identified in maize in the late 1940 s by Barbara McClintock, as mobile pieces of genetic material acting as mutable elements (McClintock, 1950). Since then a variety of different elements have been characterized and used as molecular tools. Early studies primarily worked with $A c$ and Spm transposable element systems (Wessler, 1988). 


\section{4}

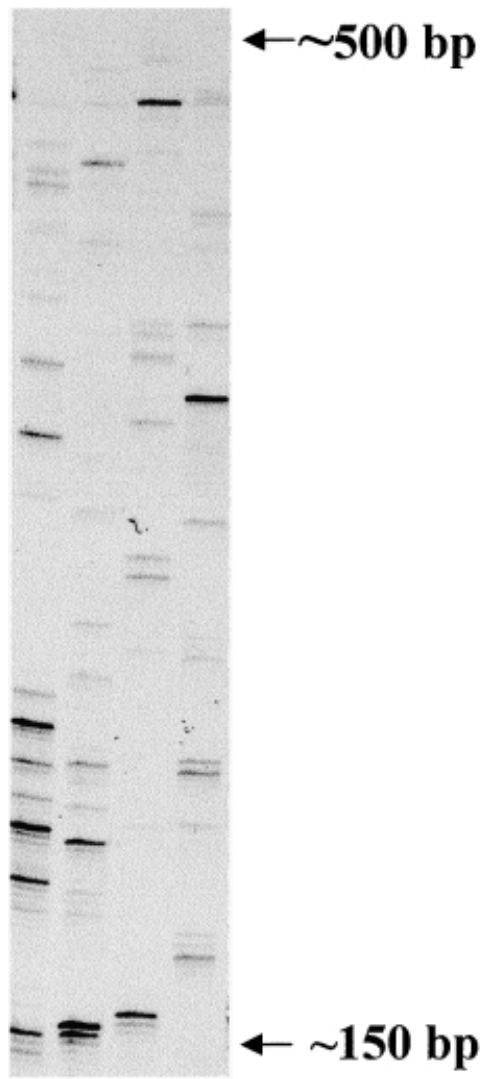

Figure 1. Analysis of amplified $M u$-tagged border fragments on $5 \%$ denaturing polyacrylamide gels.

$M u$-tagged DNA fragments were prepared from four independent $\mathrm{Mu}$ active maize lines by digestion with Mlul and Msel, followed by ligation to both a Mlul-biotinylated adaptor and a non-biotinylated Msel adaptor. $M u$-tagged fragments were selected by amplification with the ${ }^{33} \mathrm{P}$ labelled Mutator-specific primer MuB, and an Msel adaptor-specific primer. Fragments at the bottom of the gel have a size of approximately 150 bases and those at the top approximately 500 bases. Some fragments at the lower end of the gel are present as multiple bands (usually two). This is due to the incomplete addition of a T nucleotide to the $3^{\prime}$ end of the amplified fragment by the Taq DNA polymerase.

However, the Mutator $(M u)$ family of elements have since become the transposons of choice for maize gene mutagenesis, for several reasons: they are present in high copy number (10-100); they transpose via replication; and they appear to insert preferentially within and around genes, leading to a forward mutation frequency to an unlinked gene by up to 100 times greater than that observed with Ac/Spm elements (Walbot, 1992). Mu-based screens to identify transposon-tagged genes have until recently been phenotype-based, with large numbers of transposon-active plants being examined for an altered morphological or biochemical feature (McLaughlin and Walbot, 1987). Phenotype-based screens require the production of plants that are homozygous for specific
$M u$-insertions. Transposons linked genetically to the phenotype are then identified through successive generations, using molecular methods such as hybridizationbased cloning and PCR. Although essentially random in its approach, and therefore requiring large numbers of plants, this procedure has led to the cloning and characterization of several genes of agronomic importance (Bennetzen etal., 1987; Wise etal., 1996) and is still a powerful tool with which to identify genes with novel phenotypes.

More recently, site-selected transposon mutagenesis (the so-called 'gene machine'; Meeley and Briggs, 1995) has allowed the identification of plants containing insertions within specific genes (Bensen etal., 1995; Das and Martienssen, 1995). Because site-selected transposon mutagenesis is based upon genotype rather than phenotype, heterozygous $M u$ insertions can be detected, removing the need to generate homozygous plants. As an extension to the site-selected transposon mutagenesis procedure, Frey etal. (1998) reported that Mu-tagged fragments could be isolated from individual plants using PCR in conjunction with a modified adaptor-amplification protocol. This procedure has the advantage that a large number of $M u$-specific fragments can be generated without any prior knowledge of the sequence that flanks the element. Our interest in generating a public functional genomics resource for maize led us to investigate whether the procedure developed by Frey etal. (1998) could be

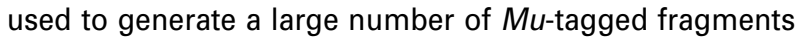
for direct sequencing. Sequence characterization of a large number of $M u$-tagged fragments would not only provide a reservoir of $\mathrm{Mu}$-tagged genes for use in future functional genomics studies, but would also help confirm or reject the hypothesis that $M u$ targets low-copy, gene-rich regions. In this report we describe both an improved method for generating large numbers of Mu-tagged fragments, and the results obtained from sequence characterization of several hundred $M u$-tagged fragments. Our results suggest that the majority of $M u$ elements do insert into low copy-number, gene-rich regions of the maize genome, and a large number of these $M u$-flanking regions show significant homology to known expressed sequence tags (ESTs) or genes.

\section{Results and Discussion}

Adaptor amplification using a modified protocol of Frey et al. (1998) produced from 35 to $50 \mathrm{Mu}$-tagged fragments per $M u$-active plant. The fragments ranged in size from 100 to $>500 \mathrm{bp}$ (Figure 1). Using genomic DNA prepared from 55 plants, sequence information was generated for 761 Mu-tagged fragments. To generate these fragments we found it necessary to modify the protocol of Frey etal. (1998). Firstly we reduced the number of amplifications before electrophoresis from two to one, and secondly we 


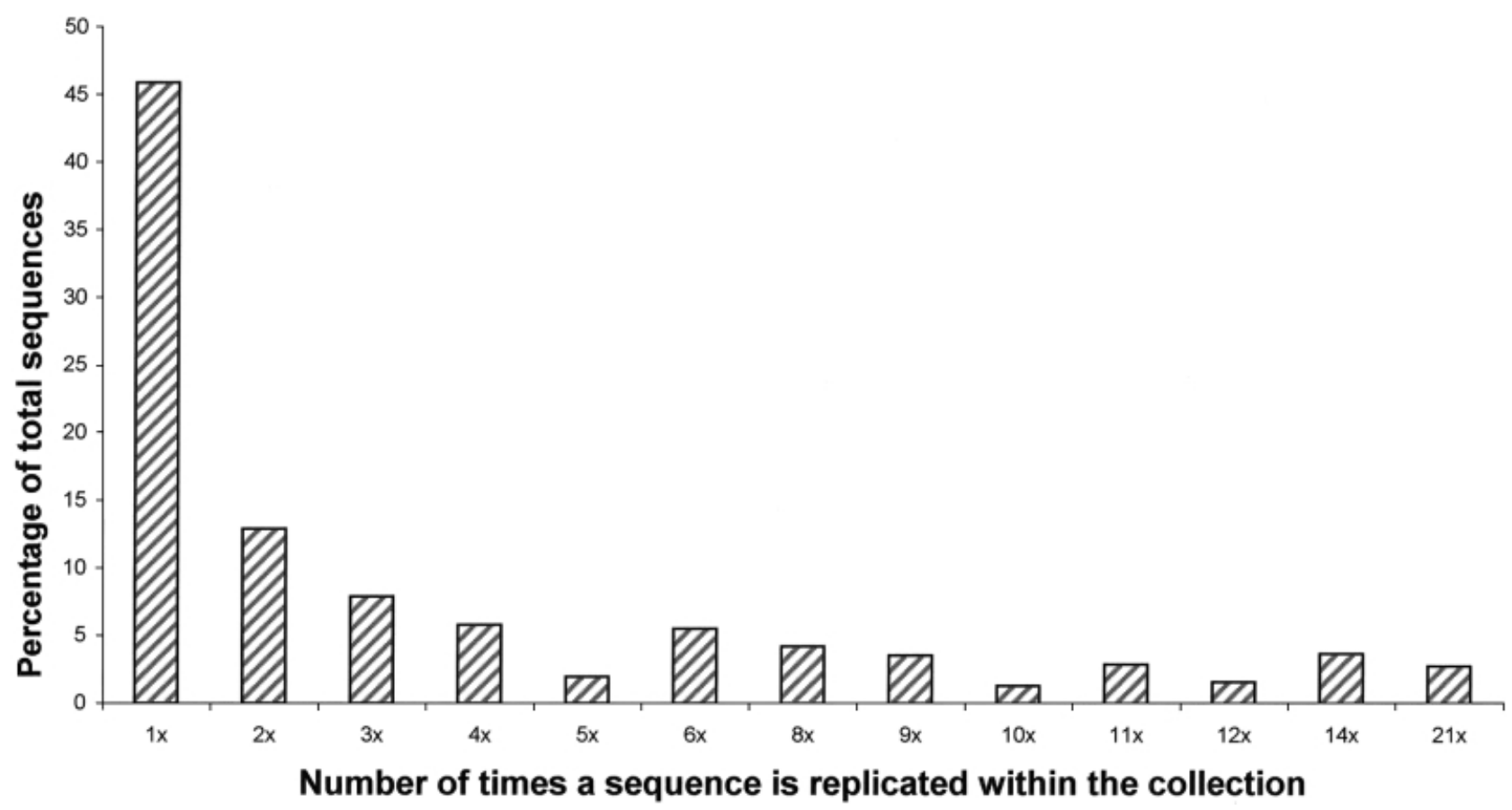

Figure 2. Representation of the number of Mu-tagged sequences present multiple times within the sequence collection.

The $761 \mathrm{Mu}$-tagged sequences generated as part of this program were compared with each other using the AUTOASSEMBLER (Perkin Elmer) software package. The number of times a sequence is replicated in the collection is presented as a histogram in which the number of times a sequence was found to be present were grouped with the numbers of sequences, as a percentage, within that category.

utilized the Mlul restriction site present in the inverted repeats of the MuDR, Mu1, Mu2 and Mu8 elements, to preselect genomic DNA enriched for $M u$-tagged fragments before amplification. In our hands, these modifications resulted in greater reproducibility of the $M u$-tagged fragment profile. However, because the modified protocol relies on the presence of a Mlul site in the inverted repeat, only $M u$-tagged elements belonging to the MuDR, Mu1, Mu2, Mu8 classes are amplified. Additionally, Mlul is sensitive to $\mathrm{CpG}$ methylation and copies of $M u$ which have undergone methylation, and may therefore be inactive and will not produce $M u$-tagged fragments by this procedure. In the work described here we chose to use Msel as the four-base recognition restriction enzyme; however, using a modified adaptor, any four- or six-base restriction enzyme which does not recognize a sequence within the $M u$ element terminal repeat could be used.

Characterization of the 761 sequences using the AUTOASSEMBLER program revealed that 389 (54\%) were represented multiple times, with some sequences being represented 21 times (Figure 2). There are two reasons why specific sequences are represented multiple times. First, the nature of the Mu transposition event (replication rather than excision) leads to parental insertions being overrepresented in the progeny when compared to new insertion events (Alleman and Freeling, 1986). Secondly, fragments within the size range amenable for both amplification and separation on denaturing polyacrylamide gels (50-600 bp) will be preferentially recovered. Removal of the duplicate sequences left 450 fragments, consisting of 350 unique sequences and 100 sequences chosen to represent those which occurred multiple times. A comparison between the size distribution of the unique and multiple sequences indicated that there was no significant difference between the two. The 450 sequences were used to screen the DNA and protein databases using both the BLASTN and BLASTX programs (Altschul et al., 1997). Using a cut-off $e$ value of $2 \mathrm{e}-04$, a total of $64(14.2 \%)$ and $48(10.6 \%)$ sequences detected putative homologous sequences in the BLASTN and BLASTX searches, respectively. 24 (5.3\%) of the sequences detected significant homologies with both BLASTN and BLASTX searches, meaning that 88 sequences $(19.5 \%)$ of the 450 unique sequences could be tentatively identified. Table 1 contains a list of the Mu-tagged fragments which had significant homology to entries within the public sequence databases. Of those sequences which showed homology to entries within the public database via the BLASTN program, $56.2 \%$ (36 out of 64 ) were either maize ESTs (produced by the maize gene discovery programme) or rice ESTs (produced by various sequencing programmes; Sasaki, 1998; http://www.zmdb.iastate.edu/ zmdb/EST_project.html). In addition to maize and rice ESTs, the BLASTN program detected homology to six entries related to either the Mu5 transposon (sequence 147) or 
Table 1. Mutator fragments with homology to entries within the public databases ${ }^{a}$

\begin{tabular}{|c|c|c|c|c|c|}
\hline Sequence ID & Plant & BLASTN hits & $e$ value & $\begin{array}{l}\text { Size } \\
\text { (bp) }\end{array}$ & $\begin{array}{l}\text { Probable } \\
\text { insertion site }\end{array}$ \\
\hline A4 & Mu3 & Zea mays Rad51B & $1 e-07$ & 241 & $5^{\prime}$ untranslated \\
\hline A17 & 101107 & C74482 Rice panicle $>3 \mathrm{~cm}$ Oryza sativa cDNA & $9 e-23$ & 317 & Intron \\
\hline A49 & kje-1 & Z. mays mRNA gs1-4 for glutamine synthetase & $1 e-67$ & 241 & Intron \\
\hline $\mathrm{C} 13$ & SH6 & Z. mays globulin-1 gene, promoter region & $6 e-05$ & 317 & Promoter \\
\hline E19 & 101206 & C28153 rice callus cDNA O. sativa cDNA & $4 e-58$ & 294 & Intron \\
\hline E38 & 101212 & Z. mays cosmid IV.1E1 $22 \mathrm{kDa}$ zein protein 21 & $2 e-08$ & 384 & Non-coding \\
\hline E40 & 101212 & Sorghum bicolor mitochondrial gene & $1 e-150$ & 286 & Coding \\
\hline E48 & 101214 & Al657302 Z. mays leaf primordia cDNA & $9 e-48$ & 439 & Coding \\
\hline E50 & 101214 & Al820302 Z. mays endosperm cDNA & $4 e-43$ & 336 & Coding \\
\hline E51 & 101214 & AW066018 Z. mays early embryo cDNA & $2 e-98$ & 290 & Coding \\
\hline F28 & 105209 & Al714888 Z. mays ear tissue cDNA & $1 e-108$ & 331 & Intron \\
\hline G12 & 105317 & Al947576.1 Z. mays stressed root cDNA & $1 e-146$ & 289 & Coding \\
\hline GF-2 & kje-1 & Al944262 Z. mays root cDNA library & $1 e-138$ & 279 & $5^{\prime}$ untranslated \\
\hline GF-7 & kje-1 & AW000013 Z. mays root cDNA library & $1 e-56$ & 304 & Coding \\
\hline H38 & 105125 & Hordeum vulgare L. (Alexis) serine carboxypeptidase & $2 e-04$ & 236 & Coding \\
\hline $\mathrm{H} 42$ & 105301 & Z. mays retrotransposon Cinful-2 & $6 e-11$ & 284 & Coding \\
\hline $\mathrm{H} 49$ & 105302 & Al665004 Z. mays endosperm cDNA & $1 e-116$ & 407 & Coding \\
\hline 111 & 105306 & Arabidopsis thaliana chromosome 5 & $4 e-29$ & 621 & unknown \\
\hline 140 & 105309 & Z. mays copia-like retrotransposon Stonor & $3 e-36$ & 674 & Intron (amidase) \\
\hline 142 & 105309 & Al737372 Z. mays ear tissue cDNA & $1 e-77$ & 394 & Intron \\
\hline 146 & 105309 & Z. mays nitrate-induced NOI protein gene & $3 e-92$ & 178 & Promoter \\
\hline 147 & 105310 & Z. mays Mu5 transposable element & $4 e-69$ & 510 & Transposon \\
\hline 155 & 105310 & Z. mays ACCase//intron containing colonist 1 and 2 & $1 e-19$ & 564 & Various retro-elements \\
\hline 163 & 105312 & Putative $26 \mathrm{~S}$ proteasome subunit athMOV34 & $2 e-09$ & 582 & $5^{\prime}$ untranslated \\
\hline 175 & 105313 & Al691241 Z. mays ear tissue cDNA library & 0.0 & 415 & Coding \\
\hline 187 & 105314 & Chromosome 5, P1 clone[Arabidopsis] & $4 e-17$ & 618 & Non-coding \\
\hline $\mathrm{J} 23$ & 105216 & Al677607 Z. mays endosperm cDNA & $3 e-58$ & 570 & Outside existing EST \\
\hline J31 & 105217 & Al783084 Z. mays root cDNA library & $1 e-49$ & 288 & Intron \\
\hline KE-6 & kje-1 & C72881 rice cDNA, partial sequence & $3 e-11$ & 363 & $5^{\prime}$ untranslated \\
\hline L37 & 105224 & Al782966 Z. mays ear tissue cDNA & $1 e-28$ & 93 & Coding \\
\hline L63 & 105103 & Z. mays $\mathrm{A} 1$ gene for $40.1 \mathrm{kDa} \mathrm{A} 1$ protein & $1 e-13$ & 168 & Intron \\
\hline L72 & 105104 & Rice mRNA for ADP-ribosylation factor & $2 e-44$ & 198 & Coding \\
\hline M7 & 105106 & AU068551 O. sativa cDNA & $2 e-07$ & 212 & Coding \\
\hline M76 & 105117 & Z. mays zeta-carotene desaturase precursor & $6 e-28$ & 148 & Coding \\
\hline N7 & DE41 & Al770851 Z. mays ear tissue cDNA & $1 e-16$ & 137 & Intron \\
\hline N22 & DE42 & Al737406 Z. mays ear tissue cDNA & $8 e-36$ & 101 & Coding \\
\hline P26 & 102304 & I33112 Sequence 3 from patent US 5589610 & $3 e-28$ & 149 & Promoter \\
\hline P65 & 103115 & Al665091 Z. mays endosperm cDNA & $1 e-46$ & 282 & Outside existing EST \\
\hline Q44 & 103123 & Z. mays PK1 gene//receptor-like protein kinase & $1 e-18$ & 256 & Intron \\
\hline Q92 & 105309 & C26026 Rice callus cDNA O. sativa cDNA & $8 e-07$ & 235 & Intron \\
\hline R9 & SH3 & C24922 Rice green shoot $O$. sativa cDNA & $4 e-08$ & 184 & Coding \\
\hline R27 & 101101 & Z. mays triosephosphate isomerase 1 gene & $2 e-22$ & 204 & $5^{\prime}$ untranslated \\
\hline R42 & 101102 & Z. mays clone Zm-Rab2-A GTP binding protein & $3 e-33$ & 192 & Coding \\
\hline R54 & 101103 & Al615233 Z. mays leaf primordia cDNA & $3 e-14$ & 166 & Intron \\
\hline R59 & 101104 & Al649598 Z. mays leaf primordia cDNA & $2 e-35$ & 233 & Coding \\
\hline R75 & 101108 & Z. mays retrotransposon Cinful- 1 & $2 e-71$ & 161 & Coding \\
\hline S2 & DE50 & AU063869 O. sativa cDNA & $1 \mathrm{e}-06$ & 382 & Outside existing EST \\
\hline S19 & 104323 & Z. mays triose phosphate/phosphate translocator & $8 e-30$ & 426 & $5^{\prime}$ untranslated \\
\hline S39 & 104319 & Al712245 Z. mays endosperm cDNA & $1 e-19$ & 315 & Intron \\
\hline S42 & 104319 & AW129804 Z. mays mixed adult tissue cDNA & $4 e-18$ & 213 & Outside existing EST \\
\hline $\mathrm{S} 80$ & 104315 & Unknown protein [A. thaliana] & $4 e-41$ & 311 & Coding \\
\hline R81 & 101108 & RICS12715A rice green shoot $O$. sativa cDNA & $9 e-07$ & 238 & Intron \\
\hline S92 & 103124 & Z. mays elF-5 gene, exons $1-2$ & $8 e-19$ & 221 & Coding \\
\hline $\mathrm{T} 25$ & 103402 & Al670571 Z. mays endosperm cDNA & $2 e-39$ & 433 & Outside existing EST \\
\hline T44 & 103405 & C20327 rice panicle at ripening stage cDNA & $2 \mathrm{e}-30$ & 302 & Outside existing EST \\
\hline T46 & 103405 & Al770425 Z. mays ear tissue cDNA & $9 e-20$ & 274 & Outside existing EST \\
\hline T53 & 103405 & Al668519 Z. mays endosperm cDNA & $7 e-37$ & 119 & $5^{\prime}$ untranslated \\
\hline T87 & 103412 & Al491543 Z. mays leaf primordia cDNA & $1 e-120$ & 300 & $3^{\prime}$ untranslated \\
\hline T90 & 103412 & AW091134 Z. mays root cDNA library & $1 e-110$ & 224 & Coding \\
\hline
\end{tabular}




\begin{tabular}{|c|c|c|c|c|c|}
\hline Sequence ID & Plant & BLASTN hits & $e$ value & $\begin{array}{l}\text { Size } \\
\text { (bp) }\end{array}$ & $\begin{array}{l}\text { Probable } \\
\text { insertion site }\end{array}$ \\
\hline U1 & 107313 & Z. mays DNA for Fd VI, complete cds & $5 e-38$ & 184 & Coding \\
\hline U14 & 107313 & Cratero-stigma plantagineum tkt7 gene for transketolase & $1 e-06$ & 120 & Coding \\
\hline U68 & 107307 & Z. mays Ama gene, RNA polymerase & $3 e-06$ & 219 & Intron \\
\hline U55 & 107308 & AU057865 O. sativa cDNA & $6 e-12$ & 243 & Outside existing EST \\
\hline 2065-34 & 2065 & Al770596 Z. mays ear tissue cDNA & $8 e-14$ & 283 & Intron \\
\hline $\mathrm{A} 4^{*}$ & Mu3 & Z. mays Rad51 & $2 e-27$ & 241 & $5^{\prime}$ untranslated \\
\hline A49* & kje-1 & Z. mays glutamine synthetase root isozyme 4 & $2 e-20$ & 241 & Intron \\
\hline C8 & $\mathrm{SH} 4$ & Putative protein $[A$. thaliana] & $1 e-10$ & 228 & Intron \\
\hline E19* & 101206 & Putative protein $[A$. thaliana] & $6 e-10$ & 294 & Intron \\
\hline $\mathrm{E} 40^{*}$ & 101212 & Hypothetical 267 kDa protein & $4 e-18$ & 286 & Coding \\
\hline F28* & 105209 & Putative protein $[A$. thaliana] & $4 e-05$ & 331 & Intron \\
\hline $\mathrm{GF}-2^{*}$ & kje-1 & O-methyltransferase [Prunus dulcis] & $1 e-10$ & 279 & $5^{\prime}$ untranslated \\
\hline H38* & 105125 & Serine carboxypeptidase II-3 precursor & $9 e-22$ & 236 & Coding \\
\hline $\mathrm{H} 42^{*}$ & 105301 & Prpol [Z. mays] & $6 e-26$ & 284 & Coding \\
\hline 18 & 105305 & Unknown protein [A. thaliana] & $9 e-30$ & 235 & Coding \\
\hline 110 & 105306 & Peroxidase $[$ A. thaliana $]$ & $1 e-05$ & 653 & Intron \\
\hline 112 & 105306 & Similarity to ANK repeat region of Fowlpox virus & $3 e-16$ & 567 & Intron \\
\hline 113 & 105306 & Hypothetical protein $[A$. thaliana] & $1 e-32$ & 516 & Coding \\
\hline 123 & 105307 & Lysosomal Pro-X carboxypeptidase-like protein & $5 e-16$ & 495 & $5^{\prime}$ untranslated \\
\hline 140 & 105309 & Putative amidase $[A$. thaliana $]$ & $9 e-22$ & 674 & Intron \\
\hline $163^{*}$ & 105312 & Putative $26 \mathrm{~S}$ proteasome subunit athMOV34 & $3 e-20$ & 582 & $5^{\prime}$ untranslated \\
\hline $175^{*}$ & 105313 & Strictosidine synthase $[A$. thaliana] & $6 e-22$ & 415 & Coding \\
\hline KE14 & $\mathrm{SH} 3$ & Human alpha-mannosidase II & $1 e-11$ & 326 & Intron \\
\hline KE18 & kje-1 & Reverse transcriptase [Ginkgo biloba] & $2 e-14$ & 202 & Coding \\
\hline L63* & 105103 & Integral membrane protein [Beta vulgaris] & $2 e-04$ & 168 & Intron \\
\hline L72* & 105104 & ADP-ribosylation factor homologue GTP-bp & $4 e-17$ & 198 & Coding \\
\hline$M 7 *$ & 105106 & Phosphatidylinositol-4-phosphate-5-kinase & $4 e-05$ & 212 & Coding \\
\hline M28 & 105109 & JM23 [Homo sapiens] & $8 e-09$ & 367 & $5^{\prime}$ untranslated \\
\hline M76* & 105117 & Zeta-carotene desaturase [A. thaliana] & $2 e-05$ & 148 & Coding \\
\hline $\mathrm{N} 22^{*}$ & DE41 & Dem [Lycopersicon esculentum] & $3 e-09$ & 107 & Coding \\
\hline P35 & 102306 & R30923-1 [H. sapiens] & $6 e-06$ & 199 & Coding \\
\hline 075 & DE47 & Receptor-like protein kinase 5 precursor & $5 e-07$ & 347 & Coding \\
\hline Q92* & DE48 & Putative amidase $[A$. thaliana] & $1 e-19$ & 235 & Intron \\
\hline R9* & $\mathrm{SH} 3$ & Contains similarity to protein phosphatase $2 \mathrm{C}$ & $1 e-21$ & 184 & Coding \\
\hline R11 & $\mathrm{SH} 3$ & 1-aminocyclopropane-1-carboxylic acid oxidase & $9 e-05$ & 138 & Coding \\
\hline R42* & 101102 & Z. mays RAS-related protein RAB-2-A & $7 e-09$ & 192 & Coding \\
\hline R56 & 101104 & Putative monosaccharide transporter 1 [Petunia] & $1 e-05$ & 239 & Intron \\
\hline $\mathrm{R} 73$ & 101105 & Putative protein $[A$. thaliana] & $1 e-09$ & 184 & Intron \\
\hline R75* & 101108 & Prpol [Z. mays] & $2 e-25$ & 161 & Coding \\
\hline S18 & 104323 & Unknown protein $[A$. thaliana] & $5 e-11$ & 431 & Intron \\
\hline S19* & 104323 & Z. mays chloroplast triose phos-translocator & $9 e-12$ & 426 & $5^{\prime}$ untranslated \\
\hline S31 & 104321 & Cinnamyl Co-A reductase $[A$. thaliana] & $2 e-05$ & 243 & Coding \\
\hline S66 & 104317 & Putative protein [A. thaliana] & $1 e-05$ & 177 & Coding \\
\hline S79 & 104315 & ATT20K18 A. thaliana DNA & $6 e-18$ & 342 & Coding \\
\hline S80* & 104315 & A. thaliana chromosome II BAC T11A7 & $2 e-04$ & 311 & Coding \\
\hline S91 & 103124 & Unknown protein $[A$. thaliana] & $1 e-41$ & 315 & Coding \\
\hline $\mathrm{T} 2$ & 104324 & ATT29A15 A. thaliana DNA & $1 e-34$ & 462 & Coding \\
\hline T20 & 103401 & T7N9.20 $[$ A. thaliana $]$ & $1 e-12$ & 287 & Intron \\
\hline T90* & 103412 & Hypothetical protein [Cicer arietinum] & $1 e-29$ & 224 & Coding \\
\hline U14* & 107313 & Transketolase 1 [Capsicum annuum] & $7 e-18$ & 120 & Coding \\
\hline U43 & 107311 & Copia-type pol polyprotein [Z. mays] & $1 e-44$ & 156 & Coding \\
\hline U57 & 107308 & Putative carbonyl reductase [A. thaliana] & $4 e-08$ & 304 & Intron \\
\hline $2065-34^{*}$ & 2065 & Hypothetical protein $[A$. thaliana] & $2 e-08$ & 283 & Intron \\
\hline
\end{tabular}

${ }^{a}$ The latest version of this table can be found at http://www.maize.bbsrc.ac.uk/mutator/random_sequencing.html.

*Sequences which also have significant BLASTN hits.

sequences associated with retro-elements, including Stoner (sequence 140); Cinful 1 (sequence R75); Cinful 2 (sequence H42); copia-type pol polyprotein (U43); and reverse transcriptase (KE18). In all six cases the $M u$ transposon had inserted into a coding region, either directly within or closely associated with the retro-element. For

(c) Blackwell Science Ltd, The Plant Journal, (2000), 22, 557-566 
$\begin{array}{llr}5 & 10 & 50 \\ \text { AGATCTACTCATCATCGAGGTGTTTTAGACACGGATTGGTATTCTAGGGA }\end{array}$

60 E $\quad G^{100}$ CTTAGGAAACGATCCTAAGCACCAGTAATTTCTAACATTTACCTCTCCAG

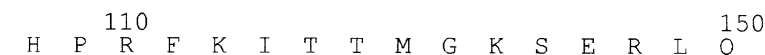

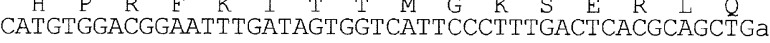
160

aaattgaacagaatcattaccactctgagaaacgtggagaaaattgagga 210

aggggaggaagatatgatcaccttgtttagggtgaattagttagccttaG 260 Y Y S 300 C
CAGTAGTACGCATAATATGAAAATGCAGGGTTGAACACATTGGTCCACTG

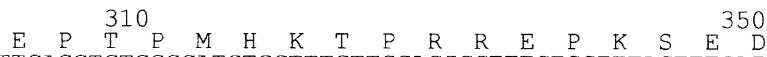
TTCAGGTGTGGGCATGTGCTTTGTTGGACGCCTTTCTGGTTTACTTTCAT

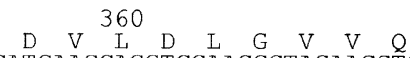

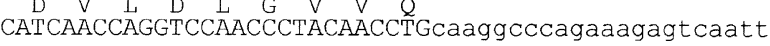
410

450 gaagttgtgatatcatgtatctgagataaatacatgcgaacgtatttctc 460

500 aattatcattactgaaaaactaatgttgaaatttagcattagttctcca 510

550 tgtcacacacacttgcaggctggcatgtgagacacacgaaacacacacac 600 640 tgggatgtgagacacacacaatagcgagttatggttgatgtctgaaatgt 650

690 tgatgcatgacacaaggcagaaatcagtcgatatgcatattccttgtaac 700 720 caaggtt tct caacctgcttcag-MUTATOR-INSERTION--gaGCTTC

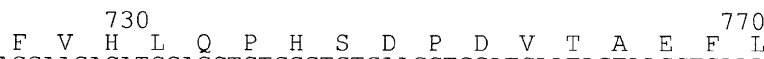
AGGAAGACATGGAGCTGTGGGTGTGAAGCTGGATCAATAGTAACCTCAAA

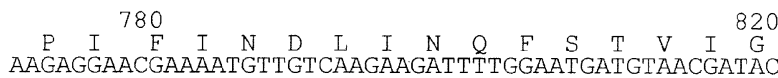

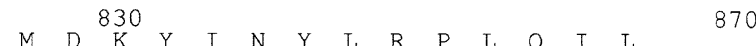
CCATTTCCTTGTACACATTATATAAGCGTGGAATCTGCAAAAGaatgtcC 880
acaaaatatatcagaaaagaataatgtaatgtttttgcactaaatatttc 880
acaaaatatatcagaaaagaataatgtaatgtttttgcactaaatatttc 930
acggggaaaaggtaacat

Figure 3. Characterization of the 140 and $092 \mathrm{Mu}$-tagged sequences. The position of the $M u$ element within the putative amidase gene and its relationship to the Stoner element are indicated. Sequences having a strong BLASTN homology ( $e$ value $=5 e-36)$ to the Stoner element are underlined (bp 1-86). Known or suspected introns within the amidase gene are in lower case (bp 150-249, 378-715, 864-939). Known or suspected exons are represented in upper case, together with the aminoacid sequence. 140 sequences (bp 1-715) are $5^{\prime}$ to the indicated Mutator insertion, whereas 092 sequences (bp 716-939) are $3^{\prime}$. In this example the non-coding strand is presented.

instance, in the case of both Cinful retro-elements the $M u$ transposon had inserted into the coding region of the Prpol gene (San Miguel etal., 1996). At the amino-acid level, sequence 140 showed homology to a putative Arabidopsis amidase (accession number AC003028; unpublished results). Further examination of the 140 sequence showed that the $M u$ element had inserted into the putative amidase gene, $3^{\prime}$ of the insertion site of a Stoner retro-element (accession number AF082134). Further sequencing also identified the second flanking region of this insertion (sequence Q92). This second sequence confirmed that in this example, the Mutator element had inserted into an intron, close to the intron-exon boundary site (Figure 3). Given that retro-elements make up most of the high copynumber repetitive DNA of the maize genome (San Miguel et al., 1996), it was surprising that $<7 \%$ (six out of 88 ) of the Mu-tagged fragments showed homology to repetitive sequences such as retro-elements. There are two possible reasons for this observation: firstly, in our protocol the choice of restriction enzymes would preclude the recovery of methylated sequences such as those known to exist in highly repetitive regions; and secondly, it has been suggested that $M u$ targets low copy sequences in generich regions (Cresse et al., 1995). Either one, or a combination of, these two possibilities would lead to the results described here. However, our observation that in all six cases examined the $M u$ element had inserted into a transcribed region associated with the retro-element does suggest that, irrespective of the copy number characteristics of the target site, $M u$ preferentially inserts into transcriptionally active DNA.

In a previous study of $16 \mathrm{Mu}$-insertion sites, Cresse et al. (1995) identified a consensus sequence present at the $M u$ insertion site. We repeated this study using all 450 unique sequences. Our results were in close agreement with the previous study (previous consensus: $5^{\prime}-\mathrm{G}-\mathrm{T}-\mathrm{T}-\mathrm{G}-\mathrm{G} / \mathrm{C}-\mathrm{A}-\mathrm{G}-$ A/G-G-3'); however, our work suggests that the consensus sequence is more flexible than previously suggested (our consensus: $5^{\prime}-\mathrm{G}-\mathrm{T} / \mathrm{C}-\mathrm{T} / \mathrm{C}-\mathrm{T} / \mathrm{G}-\mathrm{G} / \mathrm{C}-\mathrm{A}>\mathrm{T}-\mathrm{G}-\mathrm{A}>\mathrm{G}-\mathrm{G}>\mathrm{C}-3^{\prime}$ ).

To investigate if the size of the $M u$-tagged fragment had an effect on its ability to identify homologous sequences, the size of the $M u$-tagged fragments was plotted against their ability to detect homologous sequences within the public databases (Figure 4). This analysis suggested that for both BLASTN and BLASTX searches, fragments in the size range $50-400 \mathrm{bp}$ had an approximately equal chance of detecting homologous sequences. However, for fragments $>400$ bp the data suggested that there was an increased probability of detecting homologous sequences using both BLASTN and BLASTX programs. To investigate this phenomenon further, the insertion sites for each of the 88 sequences identified in the BLASTN and BLASTX searches were examined. Given the limited availability of full-length maize gene sequences, it was only possible to determine the exact location of the $M u$ insertion for 80 of the 88 BLASTN /BLASTX hits (column 6 in Table 1). The various $M u$ insertions were then divided into those that were associated with genes but not translated (promoters, 3', 5', transcribed regions and introns); and those associated with translated regions (coding regions). This study showed that $44(55 \%)$ of the insertions were within nontranslated regions, and $36(45 \%)$ were in translated 


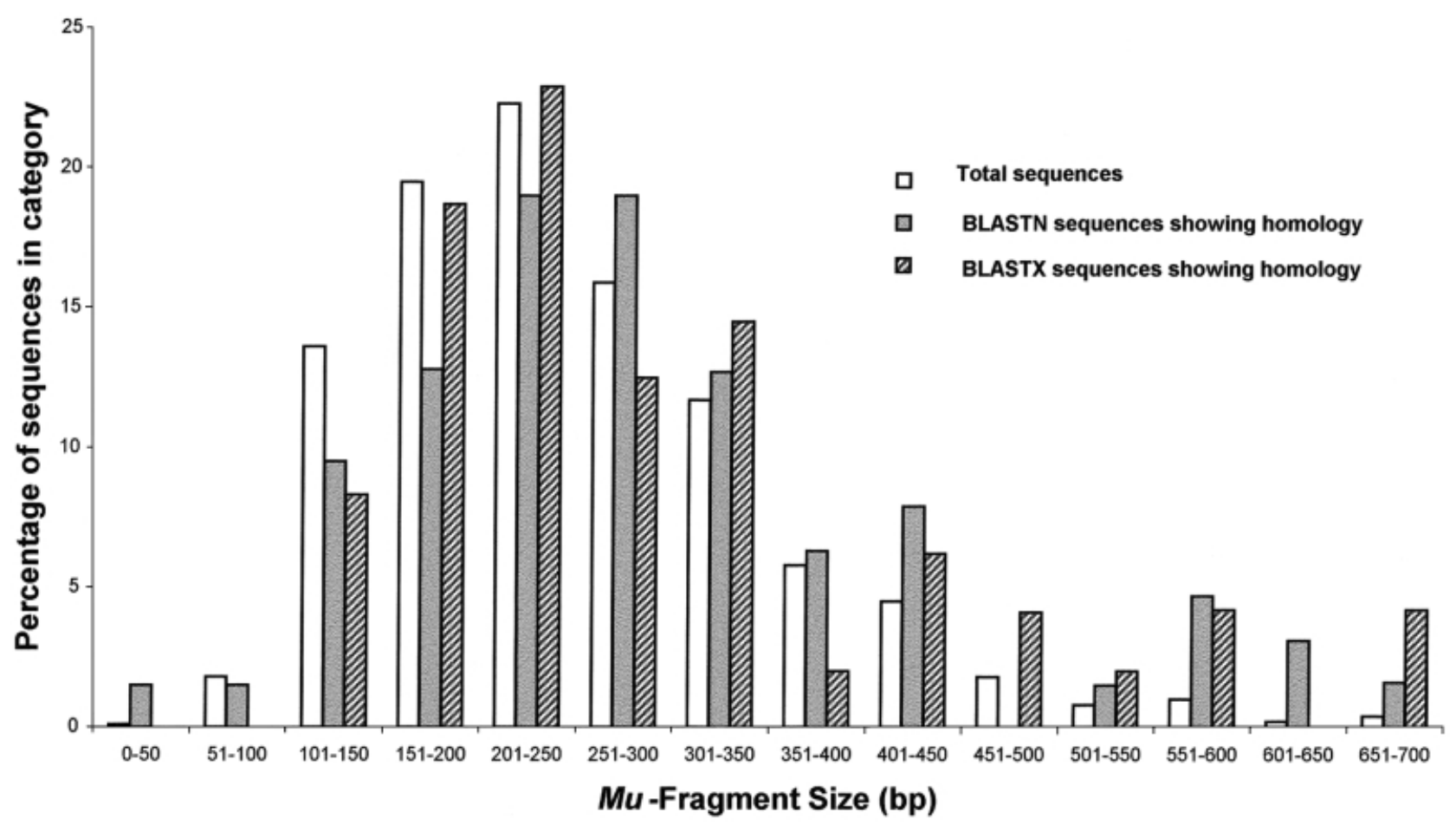

Figure 4. Comparison of the size of $M u$-tagged fragments with their ability to detect homologous sequences in the public databases.

The 450 unique sequences generated in this program were compared to sequences within the Genbank and EMBL databases (as of December 1999) via both BLASTN and BLASTX programs, using the default values. The results are presented as a histogram in which the sizes of fragments were compared to their ability to detect significant homologies.

Table 2. Maize leaf cDNAs with homology to random Mutator-tagged fragments

\begin{tabular}{lll}
$\begin{array}{l}\text { EST } \\
\text { sequence } \\
\text { ID }\end{array}$ & & $e$ value \\
\hline 1 & BLASTX hits & $1 \mathrm{e}-05$ \\
3 & LRR-like protein [Arabidopsis thaliana] & $3 \mathrm{e}-35$ \\
4 & Adenylosuccinate lyase [Escherichia coli] & $4 \mathrm{e}-44$ \\
5 & Cell division protein [Homo sapiens] ${ }^{*}$ & $8 \mathrm{e}-32$ \\
6 & ADP-ribosylation factor [Zea mays] & $4 \mathrm{e}-16$ \\
7 & Photosystem I reaction centre subunit [Z. mays] & $7 \mathrm{e}-23$ \\
$8+9$ & Nicotianamine synthase [H. vulgaris] & $4 \mathrm{e}-38$ \\
10 & Glycine-rich RNA-binding protein [Z. mays] & $2 \mathrm{e}-06$ \\
\hline
\end{tabular}

${ }^{*}$ Control sequences with known homology.

regions. This observation suggests that whilst $M u$ may target genes, within genes it does not appear have any site preference. However, if the observation that Mutator preferentially targets genes is correct, then the majority of our Mu-tagged fragments should be homologous to genes. Yet this is contrary to our observation that only $19.5 \%$ of the $M u$-tagged sequences show homology to known ESTs or genes. To confirm that a significant number of our unidentified $\mathrm{Mu}$-tagged fragments were also derived from genes, 3000 arrayed maize leaf cDNA clones were hybridized with 20 randomly chosen, uni- dentified $M u$-tagged fragments, and with two $M u$-tagged fragments previously shown to share homology with either the maize ADP-ribosylation factor (L72) or the human JM23 protein (M28). Following autoradiography, 10 hybridizing clones were identified. Sequence analysis of these clones revealed nine unique sequences. Sequence alignment of the cDNAs and the corresponding $M u$-tagged fragments showed that, while there was significant homology (ranging from 85 to $95 \%$ at the nucleotide level) between the two, in none of the nine cases examined was there $100 \%$ homology. This suggests that, while the cDNA

(C) Blackwell Science Ltd, The Plant Journal, (2000), 22, 557-566 
inserts and analogous $\mathrm{Mu}$-tagged fragments probably represent members of the same gene family, they do not represent the same genes. When used to screen the public database via the BLASTX program, all the cDNA clones showed significant homology ( $e$ value $>1 e-05$ ) to entries within the public databases (Table 2). One cDNA clone, clone 5, showed homology to a member of the maize ADPribosylation factor gene family, and another, clone 4, showed homology to the human cell-division protein, which itself has significant homology to JM23. While we had no previous information on the expression profile of the maize JM23 protein homologue, previous work by Regad etal. (1993) showed that plant ADP-ribosylation factor is expressed in leaf tissue. In all cases, except for the ADP-ribosylation factor and the JM83 sequences, the region of homology between the relatively large cDNA inserts $(1-1.5 \mathrm{~kb})$ and the sequences uncovered in the corresponding BLASTX screen did not overlap with the original $M u$-tagged fragment. It was therefore not surprising that the original $M u$-tagged fragment failed to identify the same sequences as the corresponding cDNA-BLASTX search. It was remarkable that the 20 random $M u$-tagged fragments identified as many as seven (nine hybridizing clones minus two previously known clones) cDNA clones from the 3000 clones screened. We believe that this result is possibly due to the ability of the Mu-tagged fragments to identify (via hybridization) all the members of their respective gene families which have $>85 \%$ nucleotide sequence homology. However, it is also possible that, considering the relatively small number of fragments used, screening the array with a larger and more diverse population of $\mathrm{Mu}$-tagged sequences might produce fewer hits per fragment. Whatever the reason for the relatively large number of hybridizing clones, our results suggest that screening further cDNA clones with Mu-tagged fragments could identify even more homologies. Taken together with the results from the direct sequencing, these observations suggest that eventually most of the $\mathrm{Mu}$ tagged fragments identified in this study will be confirmed as being derived from, or close to, transcribed sequences.

A fundamental principle of transposon tagging is that insertion of the element into a gene results in an inheritable phenotype. Somatic insertions have been identified as a significant problem with transposon tagging based on the Mutator system (Qin etal., 1991). To confirm the status of the $M u$ insertion described here, putative heterozygotes for insertions A49 (glutamine synthetase 1-4); KE14 (alpha mannosidase II); M76 (zetacarotene desaturase precursor); 075 (receptor-like protein kinase 5 precursor); and U14 (transketolase) were backcrossed with the inbred line B73 for four generations. In all cases, B73 was used as the pollen source. At each generation, the transmission of the specific $M u$ insertion was monitored via PCR using an insertion-specific primer and the MuB primer. In all cases, Mu-tagged fragments were transmitted at or near the expected frequency $(50: 50$; data not shown). These observations confirmed that the original $M u$ insertions were germinal in origin. We believe that the reason lies in our strategy of isolating individual $M u$ bands from denaturing polyacrylamide gels. We believe that distinct $M u$ bands isolated from the gel were more likely to be germinal insertions, whereas somatic insertions were more likely to be represented by the faint background smear present on the polyacrylamide gel.

Following four generations of back-crossing to B73, amplification-positive plants were selfed and the progeny examined for any obvious phenotype. From the five separate insertions examined, only M76 (zeta-carotene desaturase precursor) had an obvious phenotype. As previously described, this zeta-carotene desaturase $\mathrm{Mu}$ tagged line produced weakly viviparous kernels. Further characterization of this line has confirmed that it represents a new allele of the vp5 phenotype (Wurtzel, 1992).

The method for generating Mu-tagged DNA fragments described here is relatively simple to perform. Our results suggest that it is amenable to being scaled up to include several thousand $M u$-active maize plants. Significantly, our results also suggest that the majority of these sequences will represent germinal insertion events. Sequence analysis of such a large number of germinally derived $M u$-tagged fragments, derived from independent plants, has the potential to reduce the bottleneck in the elucidation of gene function.

\section{Experimental procedures}

\section{Growth and maintenance of maize plants}

Mutator active maize seed in a W22 background was a kind gift of Dr Martienssen of the Cold Spring Harbor Laboratory. Plants were grown as described by Neuffer (1993). Mu-active plants were maintained as heterozygote families by random mating within the $M u$-active pool. Southern blot hybridization (Southern, 1975) of DNA prepared from this material indicated that individual plants contained between 35 and $50 \mathrm{Mu}$ elements when probed with a ${ }^{32}$ P-labelled $M u$-inverted repeat (Maniatis et al., 1982).

\section{Isolation and characterization of Mu-tagged DNA fragments}

$M u$-tagged fragments were isolated by a modification of the method of Frey et al. (1998). Briefly, $100 \mathrm{ng}$ of total genomic DNA was digested with 2 units of Mlul (a restriction enzyme with the six-base recognition sequence A_CGCGT) and 4 units of Msel (a restriction enzyme with the four-base recognition sequence T_TAA) in a volume of $50 \mu \mathrm{l}$ for $1 \mathrm{~h}$ at $37^{\circ} \mathrm{C}$. Following the restriction digestion, $50 \mathrm{ng}$ of a biotinylated M/ul adapter (consisting of a biotinylated 17mer: biotin-5'-CTCGTAGACTGCGTAAC- $3^{\prime}$ and a complementary 15mer: 5'-CGCGGTTACGCAGTC-3') and $50 \mathrm{ng}$ of a non-biotinylated Msel adaptor (consisting of a 16mer: 5'-GACGATGAGTCCTGAG-3' and a complementary 
14mer: $5^{\prime}$-TACTCAGGACTCAT-3'), together with $5 \mu 10 \mathrm{~mm}$ ATP and 1 unit $T_{4}$ DNA ligase, were added to the digestion mix to make a total volume of $60 \mu \mathrm{l}$. The ligation was allowed to proceed for $2 \mathrm{~h}$ at $37^{\circ} \mathrm{C}$. Genomic fragments linked to the Mlul adaptors were isolated and purified using $10 \mu \mathrm{l}$ Dynal (NY, USA) magnetic streptavadin beads (Tong and Smith, 1992.). Following purification (Mathes et al., 1998;), a $1 \mu$ l aliquot of the DNA-bead complex was subjected to amplification using a $\left[{ }^{33} \mathrm{P}\right] 5^{\prime}$-labelled primer specific for a conserved region of the Mutator inverted repeat $\left(5^{\prime}\right.$ CAGAATTCCATAATGGCAATTATCTC- $3^{\prime}$ ) and a primer specific for the $M$ sel adaptor (5'-GACGATGAGTCCTGAGTAA-3') in $25 \mu \mathrm{l}$ with 1 unit of Taq DNA polymerase, using the cycling conditions previously described by Mathes et al. (1998). Following amplification the entire reaction was electrophoresed in a $5 \%$ denaturing polyacrylamide gel. Individual bands were visualised via autoradiography and those $>50$ bp excised and eluted as described by Maniatis etal. (1982). Individual bands were then re-amplified using the original Mlul and Msel primers, and subjected to direct sequencing using the Perkin Elmer (Cheshire, UK) Applied Biosystems BIG DYE terminator kit and the original Msel-specific primer.

\section{Screening plasmid cDNA libraries with Mu-tagged fragments}

$50 \mathrm{ng}$ each of 20 randomly chosen $M u$-tagged fragments which did not have homology to sequences within the databases, one Mu-tagged fragment with homology to the maize ADP-ribosylation factor, and one $M u$-tagged fragment with homology to the human cell-division protein JM23, were pooled and labelled with $\alpha\left[{ }^{32} \mathrm{P}\right] \mathrm{dCTP}$ according to the method of Feinberg and Vogelstein (1983). The labelled fragments were used to probe 3000 arrayed colonies containing plasmid with cDNAs derived from maize leaf mRNA. Following hybridization at $65^{\circ} \mathrm{C}$ in $6 \times \mathrm{SSC}, 5 \times$ Denhardt's and $2 \%$ SDS for $16 \mathrm{~h}$, the filters were washed three times at $65^{\circ} \mathrm{C}$ in $0.2 \times$ SSC, $1 \%$ SDS. The washed filters were subjected to autoradiography with a single intensifying screen for 3 days at $70^{\circ} \mathrm{C}$. Hybridizing clones were purified to single colonies and used to prepare plasmid DNA. Purified plasmids were sequenced using the Perkin Elmer Applied Biosystems BIG DYE terminator kit and the universal forward M13 primer.

\section{Database screening}

Primer and vector sequences were removed using AUTOASSEMBLER (Perkin Elmer). Corrected sequences were screened against all of the GenBank and EMBL databases using both BLASTN and BLASTX programs (Altschul etal., 1997). Both BLASTN and BLASTX programs were used with the default settings. Only homologies with an $e$ value $>2 \mathrm{e}-04$ were recorded as being significant.

\section{Acknowledgements}

IACR-Long Ashton receives grant-aided support from the Biotechnology and Biological Sciences Research Council of the United Kingdom. S. Hanley was funded by a Zeneca Plant Science award to K.J.E.; S. Haines was funded by a fruit and cider studentship in collaboration with Advanta; M. Hegarty was funded by an Industrial CASE studentship with Zeneca Plant Sciences; D. Stevenson was funded by a BBSRC Agri-Foods award; and D. Edwards was funded by the Framework IV programme ZEROPA. Seed for the various $M u$ insertions described here are available for research applications by writing to Keith J. Edwards.

\section{References}

Alleman, M. and Freeling, M. (1986) The Mu transposable elements of maize: evidence for transposition and copy number regulation during development. Genetics, 112, 107-119.

Altschul, S.F., Madden, T.L., Schaffer, A.A., Zhang, J., Zhang, Z., Miller, E.W. and Lipman, D.L. (1997) Gapped BLAST and PSIBLAST: a new generation of protein database search programs. Nucl. Acids Res. 25, 3389-3402.

Bennetzen, J.L., Cresse, A., Brown, W.E. and Lee, L. (1987) Molecular cloning of maize genes by transposon tagging with Mutator. In Plant Gene Systems and their Biology, UCLA Symposia on Molecular and Cellular Biology New Series, Vol. 62 (Key, J.L. and McIntosh, L., eds). New York: Alan R. Liss, pp. 183-204.

Bensen, R.J., Johal, G.S., Crane, V.C., Tossberg, J.T., Schnable, P.S., Meeley, R.B. and Briggs, S.P. (1995) Cloning and characterization of the maize An1 gene. Plant Cell, 7, 75-84.

Cresse, A.D., Hulbert, S.H., Brown, W.E., Lucas, J.R. and Bennetzen, J.L. (1995) Mu1-related transposable elements of maize preferentially insert into low copy number DNA. Genetics, 140, 315-324.

Das, L. and Martienssen, R.A. (1995) Site-selected mutagenesis at the Hcf106 locus in maize. Plant Cell, 7, 287-294.

Edwards, K.J., Veuskens, J., Rawles, H., Daly, A. and Bennetzen, J.L. (1996) Characterization of four dispersed repetitive DNA sequences from Zea mays and their use in constructing contiguous DNA fragments using YAC clones. Genome, 39, 811-817.

Feinberg, A.P. and Vogelstein, B. (1983) A technique for radiolabeling DNA restriction endonuclease fragments to high specific activity. Anal. Biochem. 132, 6-13.

Frey, M., Stettner, C. and Gierl, A. (1998) A general method for gene isolation in tagging approaches amplification of insertion mutagenised sites (AIMS). Plant J. 13, 717-721.

Gaut, B.S. and Doebley, J.F. (1997) DNA sequence evidence for the segmental allotetraploid origin of maize. Proc. Natl Acad. Sci. USA, 94, 6809-6814.

Maniatis, T., Fritsch, E.F. and Sambrook, J. (1982) Molecular Cloning: A Laboratory Manual. New York: Cold Spring Harbor Laboratory.

Mathes, M., Daly, A. and Edwards, K.J. (1998) Amplified fragment length polymorphisms. In Molecular Tools for Screening Biodiversity: Plants and Animals (Karp, A., Isaac, P.G. and Ingram, D.S., eds). London: Chapman \& Hall, pp. 181-192.

McClintock, B. (1950) The origin and behaviour of mutable loci in maize. Proc. Natl Acad. Sci. USA, 36, 344-355.

McLaughlin, M. and Walbot, V. (1987) Cloning of the Bronze2 locus of maize by transposon tagging and differential hybridisation. Genetics, 117, 771-776.

Meeley, R.B. and Briggs, S.P. (1995) Reverse genetics for maize. Maize Genet. Newsl. 69, 67-82.

Neuffer, M.G. (1993) Growing maize for genetic studies. In The Maize Handbook (Freeling, M. and Walbot, V., eds). New York: Springer Verlag, pp. 197-208.

O'Sullivan, D., Edwards, D. and Edwards, K.J. (2000) Maize Genomics. Agbiotechnet, 2, 1-6.

Qin, M., Robertson, D.S. and Ellinboe, A.H. (1991) Cloning of the Mutator transposable element MuA2: a putative regulator of somatic mutability of the a1-Mum2 allele in maize. Genetics, 129, 845-854. 
Regad, F., Bardet, C., Tremousaygue, D., Moisan, A., Lescure, B. and Axelos, M. (1993) cDNA cloning and expression of an Arabidopsis GTP-binding protein of the ARF family. FEBS Lett. 316, 133-136.

San Miguel, P., Tikhonov, A., Jin, Y.K. et al. (1996) Nested retrotransposons in the intergenic regions of the maize genome. Science, 274, 765-768.

Sasaki, T. (1998) The rice genome project in Japan. Proc. Natl Acad. Sci. USA, 95, 2027-2028.

Southern, E.M. (1975) Detection of specific sequences among DNA fragments separated by gel electrophoresis. J. Mol. Biol. 98, 503-517.

Tong, X. and Smith, L.M. (1992) Solid phase method for the purification of DNA sequencing reactions. Anal. Chem. 64, 2672-2677.

Walbot, V. (1992) Strategies for mutagenesis and gene cloning using transposon tagging and T-DNA insertional mutagenesis. Annu. Rev. Plant Phys. Plant Mol. Biol. 43, 49-82.

Wessler, S.R. (1988) Phenotypic diversity mediated by the maize transposable elements Ac and Spm. Science, 242, 399-405.

Wise, R.P., Dill, C.L. and Schnable, P.S. (1996) Mutator-induced mutations of the rf1 nuclear fertility restorer of T-cytoplasm maize alter the accumulation of T-urf13 mitochondrial transcripts. Genetics, 143, 1383-1394.

Wurtzel, E.T. (1992) Use of a Ds chromosome-breaking element to examine maize $v p 5$ expression. J. Hered. 83, 109-113. 